\title{
27
}

\section{RELIABILITY ANALYSIS AND OPTIMAL DESIGN OF MONOLITHIC VERTICAL WALL BREAKWATERS}

\author{
J.D. Sørensen, H.F. Burcharth \& E. Christiani \\ Aalborg University, Sohngaardsholmsvej 57, DK-9000 Aalborg, Denmark
}

\begin{abstract}
Reliability analysis and reliability-based design of monolithic vertical wall breakwaters are considered. Probabilistic models of the most important failure modes, sliding failure, failure of the foundation and overturning failure are described. Relevant design variables are identified and reliability-based optimization problems are formulated. Results from an illustrative example are given.
\end{abstract}

\section{Introduction}

Coastal structures are normally designed on a deterministic basis using level 1 codes. However, the design wave which defines the dominating load on the structures is estimated on a probabilistic basis, for example as the characteristic significant wave height having a return period of 100 years. All other uncertainties are taken into account using safety factors or partial safety factors.

Breakwater structures are used under quite different conditions. The expected lifetime can be from 5 years (interim structure) to 100 years (permanent structure) and the accepted level of probability of failure in the expected lifetime can vary from a very small number, e.g. $10^{-4}$ if failure of the breakwater results in significant damage to large probabilities, e.g. 0.5 if the consequences are insignificant, see Burcharth [1]. Further a number of serious failures of breakwaters have been reported during the last 20 years. In order to obtain more rational and consistent estimates of the reliability of breakwater structures and in order to be able to perform a reliability-based design optimization, the paper describes a probabilistic model of the failure modes of typical vertical wall breakwaters and of the uncertainties related to these failure modes.

As an example monolithic vertical wall breakwaters built of reinforced concrete caissons filled with sand are considered. Important failure modes for this type of breakwaters are identified, namely sliding failure, failure of the foundation and overturning failure. Limit state functions are formulated and stochastic models for the uncertain variables are identified in section 2. Next reliability-based design optimization of breakwaters are considered in section 3 . Results of a reliability analysis and a reliability-based design of a monolithic vertical wall breakwater are presented in section 4. 


\section{Failure modes for monolithic vertical wall breakwaters}

Vertical wall breakwaters can be constructed in a number of different ways. The most common types are shown in figure $1 \mathrm{a}$ and $1 \mathrm{~b}$, namely one type where the caisson is placed on a thin bedding layer and one type where the caisson is placed on a rubble mound.

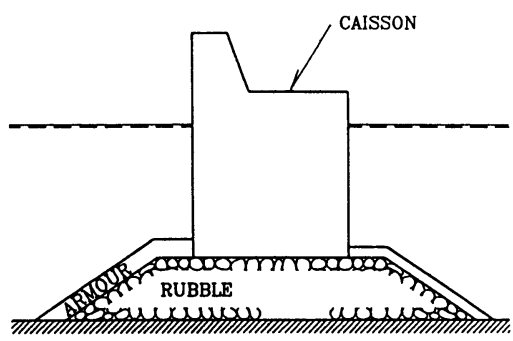

CLAY, SAND OR ROCK

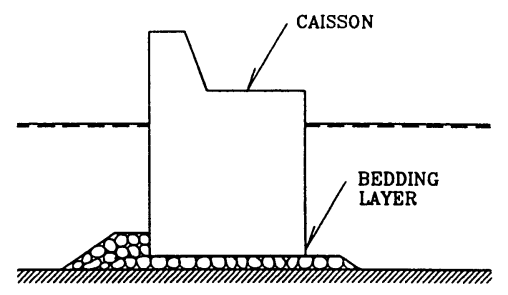

CLAY, SAND OR ROCK

Figure 1a and 1b. Main types of vertical wall breakwaters.

Modelling of wave load by the Goda model

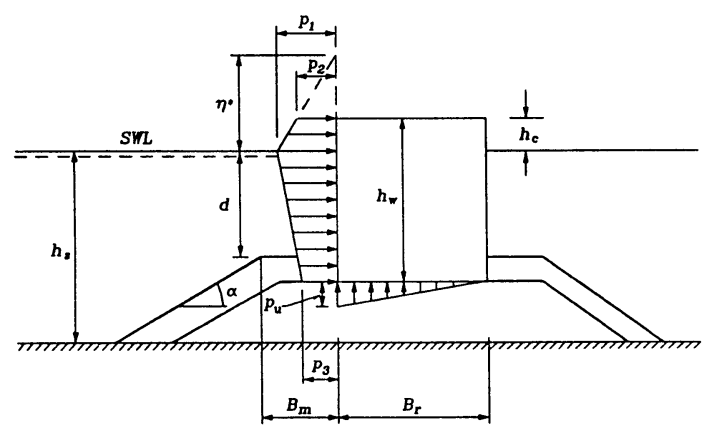

Figure 2. Wave induced pressure under a wave crest modelled by the Goda model.

Models for estimating the wave load on vertical wall breakwaters have been developed by Goda [2] and Goda et al. [3]. The wave load is used to formulate safety margins for the most important failure modes for vertical wall breakwaters, namely sliding, foundation failure and overturning. Failure of the caisson structure and hydraulic stability failure of the armour layer and mound are not considered in this paper.

Figure 2 shows the wave pressure model suggested by Goda for the case of a conventional vertical front where wave breaking is not enhanced by a steep sea bed or structural configurations. The model is based on model tests and includes the effect of breaking waves to the extent of normal accidental (non-provoked) wave breaking. A detailed description is given in Goda [4] and Burcharth et al. [5]. $p_{1}, p_{2}, p_{3}$ and $p_{u}$ in figure 2 are

$$
\begin{aligned}
& p_{1}=0.5(1+\cos \beta)\left(\alpha_{1}+\alpha_{2} \cos ^{2} \beta\right) \rho_{w} g H_{d} \\
& p_{3}=\alpha_{3} p_{1}
\end{aligned}
$$




$$
\begin{aligned}
& p_{2}= \begin{cases}\left(1-\frac{h_{c}}{\eta^{*}}\right) p_{1} & \text { for } \eta^{*}>h_{c} \\
0 & \text { for } \eta^{*} \leq h_{c}\end{cases} \\
& p_{u}=0.5(1+\cos \beta) \alpha_{1} \alpha_{3} \rho_{w} g H_{d}
\end{aligned}
$$

where $\eta^{*}=0.75(1+\cos \beta) H_{d}, \beta$ is the angle of incidence of waves, $\rho_{w}$ is the mass density of sea water, $H_{S}$ is the significant wave height, $H_{d}$ is the wave height to be used in the Goda formula defined as the highest wave in the design sea state just in front of the breakwater. $H_{d}$ is taken as $1.8 H_{S}$, see Goda [4]. However, $H_{d}$ cannot be larger than the wave breaking height, see [4]. $\alpha_{1}=\alpha_{1}\left(H_{S}, d, h_{s}, L\right), \alpha_{2}=\alpha_{2}\left(H_{S}, d, h_{s}, L\right)$, $\alpha_{3}=\alpha_{3}\left(H_{S}, d, h_{s}, h_{c}, L\right)$, see [4] and $L$ is the wave length corresponding to that of the significant wave.

On the basis of the wave pressures defined by $p_{1}, p_{2}, p_{3}$ and $p_{u}$ and the tidal elevation $\zeta$ the resultant horisontal force $F_{H}=F_{H}\left(H_{S}, \zeta, d, h_{s}, h_{c}, B_{r}\right)$ and the vertical (uplift) force $F_{U}=F_{U}\left(H_{S}, \zeta, d, h_{s}, h_{c}, B_{r}\right)$ can be determined. Also the corresponding resultant moments about the heel of the caisson can be determined : $M_{H}=M_{H}\left(H_{S}, \zeta, d, h_{s}, h_{c}, B_{r}\right)$ and $M_{U}=M_{U}\left(H_{S}, \zeta, d, h_{s}, h_{c}, B_{r}\right)$. It should be noted that the Goda model can be modified to take into account impulsive forces, see Takahashi et al. [6].

\section{Sliding failure}

Stability failure by sliding can be modelled by the limit state function

$$
g=\left(F_{G}-Z F_{U}\right) \mu-Z F_{H}
$$

where $\mu$ is the coefficient of friction between the bedding layer and the caisson, $F_{G}=$ $F_{G}\left(\zeta, h_{w}, B_{r}\right)$ is the weight of the caisson reduced for boyancy and $Z$ is a stochastic variable modelling the model uncertainty connected with the Goda model, see van der Meer et al. [7].

\section{Overturning failure}

Failure by overturning can be modelled by the limit state function

$$
g=M_{G}-Z M_{U}-Z M_{H}
$$

where $M_{G}=M_{G}\left(\zeta, h_{w}, h_{c}, B_{r}\right)$ is the moment of the weight of the caisson about the heel.

\section{Foundation failure}

If it is assumed that the soil consists of clay only, figure 3 shows some of the most important kinematic admissible foundation failure modes corresponding to the two types of structures shown in figure 1. $F_{R}$ is the resultant wave load from $F_{G}$ and $F_{H}$. As an example a limit state function is described in the following for foundation failure by rotation of the caisson placed on a bedding layer. Figure 4 describes the rotation mechanism. The centre of rotation is $O\left(x_{0}, z_{0}\right)$. The failure mechanism is based on the assumption of plane strain conditions in the soil, i.e. a two-dimesional solution for the equilibrium of the considered slip surface in the soil. Note that it is assumed that tensile stresses cannot occur under the caisson base plate. 


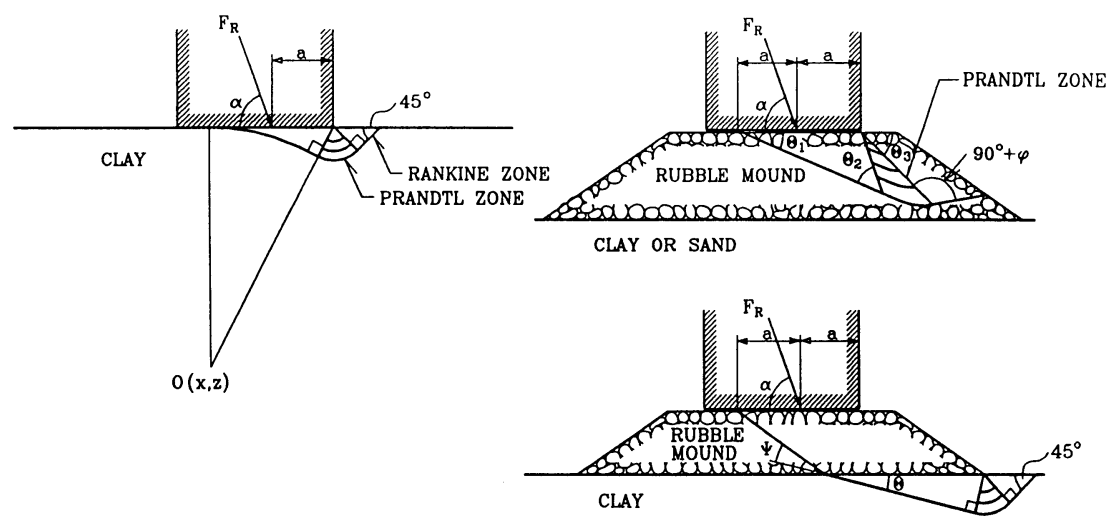

Figure 3. Foundation failure mechanisms.

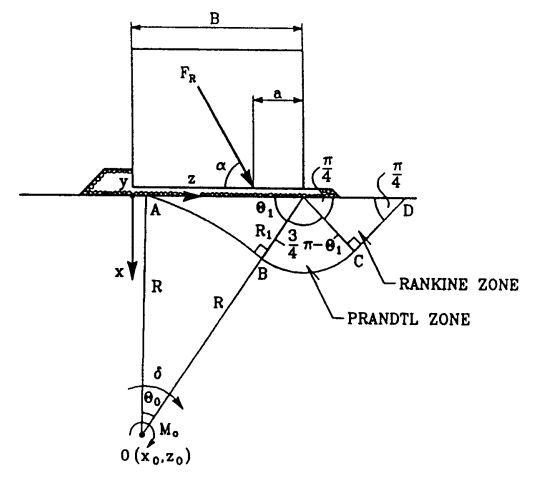

Figure 4. Rotation mechanism.

The increment of the external work $W_{E}$ is calculated as the moment from the pressures defined in figure 2 around the centre of rotation $O\left(x_{0}, z_{0}\right)$ multiplied by a unit increment of the rotation angle $\delta$.

The total increment of the internal work $W_{I}$ covers the rim of all fracture zones and the contribution from the Prandtl and Rankine fracture zones, i.e. $W_{I}=W_{1}+W_{2}+W_{3}+$ $W_{4}+W_{5}$ where $W_{1}, W_{2}$ and $W_{3}$ are the works along the rim of the fracture zones $A B$, $B C$ and $C D . W_{4}$ and $W_{5}$ are the internal works in the Prandtl zone and in the Rankine zone.

Internal work along the rim of the fracture zones can be calculated from the point of rotation $O\left(x_{0}, z_{0}\right)$ :

$$
W_{1}=\delta R^{2} \int_{0}^{\theta_{o}} c_{u}(\theta) d \theta \quad W_{2}=\delta R R_{1} \int_{0}^{\frac{3 \pi}{4}-\theta_{1}} c_{u}(\theta) d \theta \quad W_{3}=\delta R \int_{0}^{R_{1}} c_{u}(a) d a
$$

where $c_{u}$ is the undrained shear strength of the clay. $R, R_{1}, \theta_{0}$ and $\theta_{1}$ are defined in 
figure 4. The internal work from the Prandtl and Rankine fracture zones are

$$
W_{4}=\delta R_{1} \int_{0}^{R_{1}} \int_{0}^{\frac{3 \pi}{4}-\theta_{1}} c_{u}(a, \theta) d \theta d a \quad W_{5}=\delta \int_{0}^{R_{1}} \int_{0}^{R_{1}-a} c_{u}(a, l) d l d a
$$

The rotation point is determined as the point where the ratio between total internal work $W_{I}$ and external work $W_{E}$ is a minimum. In the reliability analysis it is taken into account that the rotation point $O\left(x_{0}, z_{0}\right)$ is dependent on the stochastic variables modelling the loading and the strength of the soil.

The undrained shear. strength of the clay is modelled as a log-Gaussian stochastic field $\left\{c_{u}(x, z)\right\}$, see e.g. Keaveny et al. [8] and Andersen et al. [9]. If only uncertainty related to $c_{u}$ is considered and the correlation lengths for $\left\{c_{u}(x, z)\right\}$ are small compared to the integration intervals it follows from the central limit theorem that the total internal work can be approximated by a normal distributed stochastic variable $W_{I}$ with mean value $\mu_{W_{I}}$ and standard deviation $\sigma_{W_{I}}$. The limit state function is written

$$
g=\mu_{W_{I}}+u_{W} \sigma_{W_{I}}-W_{E}=\sum_{i=1}^{5} E\left[W_{R i}\right]+u_{W} \cdot\left(\sum_{i=1}^{5} \sum_{j=1}^{5} \operatorname{Cov}\left[W_{R i}, W_{R j}\right]\right)^{\frac{1}{2}}-W_{E}
$$

where $u_{W}$ is a realization of a normal distributed stochastic variable $U_{W}$ with mean 0 and unit standard deviation.

Further e.g. the mean value of $W_{4}$ is

$$
E\left[W_{4}\right]=\delta R_{1} \int_{0}^{R_{1}} \int_{0}^{\frac{3 \pi}{4}-\theta_{1}} E\left[c_{u}(a, \theta)\right] d \theta d a
$$

where $E\left[c_{u}(a, \theta)\right]$ is the expected value of $c_{u}$ at the position described by $(a, \theta)$ and the variance of $W_{5}$ is

$$
\operatorname{Var}\left[W_{5}\right]=\delta \int_{0}^{R_{1}} \int_{0}^{R_{1}-a_{1}} \int_{0}^{R_{1}} \int_{0}^{R_{1}-a_{2}} \operatorname{Cov}\left[c_{u}\left(a_{1}, l_{1}\right), c_{u}\left(a_{2}, l_{2}\right)\right] d l_{2} d a_{2} d l_{1} d a_{1}
$$

where $\operatorname{Cov}\left[c_{u}\left(a_{1}, l_{1}\right), c_{u}\left(a_{2}, l_{2}\right)\right]$ is the covariancefunction of $c_{u}$ at the positions corresponding to $\left(a_{1}, l_{1}\right)$ and $\left(a_{2}, l_{2}\right)$.

\section{General formulation of optimization (design) problem}

In design of breakwaters the main objectives are usually that the breakwaters are as inexpensive as possible and have a satisfactory reliability. These two contradictory requirements can be treated by formulating reliability-based optimization problems on a decision-theoretical basis, see Enevoldsen \& Sørensen [10]. The design (decision) variables are denoted $\mathbf{b}=\left(b_{1}, \ldots, b_{N}\right)$, i.e. the number of design variables is $N$. The design variables can e.g. be the height and the width of the breakwater, see below. If the 
objective function is chosen as the total expected costs $C_{T}$ of the structure during the lifetime the optimal design can be found as the solution to the optimization problem

$$
\begin{array}{ll}
\min _{\mathbf{b}} & C_{T}(\mathbf{b})=C_{I}(\mathbf{b})+C_{F} P_{F}(\mathbf{b}) \\
& b_{i}^{l} \leq b_{i} \leq b_{i}^{u} \quad, i=1, \ldots, N
\end{array}
$$

where $b_{i}^{l}$ and $b_{i}^{u}$ are lower and upper bounds to $b_{i} . C_{I}$ is the initial/construction costs, $C_{F}$ is the costs of failure and $P_{F}$ is the probability of failure during the expected lifetime of the breakwater. If more than one failure mode has to be considered then failure of the structure has to be modelled by a system for which $P_{F}$ can be estimated using First Order Reliability Methods (FORM), see Madsen et al. [11]. If only one failure mode is considered then $P_{F}=\Phi(-\beta)$ where the reliability index $\beta$ is evaluated by FORM on the basis of a limit state function $g(\mathbf{x})=0$ where $\mathbf{x}$ is a realisation of the stochastic variables $\mathbf{X}=\left(X_{1}, \ldots, X_{n}\right) \Phi$ is the standard normal distribution function.

In the optimization problem (12)-(13) design (decision) variables and costs related to inspection, maintenance and repair can be included, see [10]. The optimization problem (12)-(13) can alternatively be formulated as an optimization problem with reliabilitybased constraints.

Generally the reliability requirements for different failure modes are not the same. Therefore it is of interest to consider the element reliability-index based optimization problem

$$
\begin{array}{clc}
\underset{\mathbf{b}}{\min } & C_{I}(\mathbf{b}) & \\
\text { s.t. } & \beta_{i}(\mathbf{b}) \geq \beta_{i}^{\text {min }} & , i=1, \ldots, M \\
& B_{i}(\mathbf{b}) \geq 0 \quad, i=1, \ldots, m \\
& b_{i}^{l} \leq b_{i} \leq b_{i}^{u} \quad, i=1, \ldots, N
\end{array}
$$

where $\beta_{i}$ is the reliability index for failure mode $i$ and $\beta_{i}^{\text {min }}$ is the corresponding lower bound on the reliability index. The above optimization problems are usually non-linear and non-convex. The optimization problems can be solved effectively using non-linear optimization algorithms and FORM, see [10]. The reliability indices in (25) are determined on the basis of limit state functions written as $g_{i}(\mathbf{x}(\mathbf{b}), \mathbf{b})=0, i=1, \ldots, M$.

In a traditional deterministic design the design (optimization) problem the constraint (15) is exchanged by the deterministic constraint

$$
B_{i}(\mathbf{b})=g_{i}\left(\mathbf{x}^{D}(\mathbf{b}, \boldsymbol{\gamma}), \mathbf{b}\right) \geq 0 \quad, i=1, \ldots, M
$$

where $\mathbf{x}^{D}$ are design values calculated using the statistical parameters for the stochastic variables $\mathbf{X}$ and e.g. partial safety factors $\boldsymbol{\gamma}$. Typically the design variables $\mathbf{b}$ will be related to the expected value of the stochastic variables.

Examples of design variables for vertical wall breakwaters are the main geometrical quantities defining the structures shown in figure 1. 


\section{Example}

A vertical wall breakwaters placed on a bedding layer is considered, see figure 1 . The following data are used: $h_{s}=13 \mathrm{~m}, d=9.5 \mathrm{~m}, h_{c}=5.5 \mathrm{~m}, h_{w}=17$ and $\rho_{w}=$ 1. The tidal elevation $\zeta$ is modelled as a stochastic variable with distribution function $F_{\zeta}(\zeta)=-\frac{1}{\pi} \arccos \left(\frac{\zeta}{\zeta_{0}}-1\right)-1$ where $\zeta_{0}=0.75 \mathrm{~m}$. The average mass density of the caisson $\rho_{c}$ including sand ballast is assumed to be normal distributed with mean value $2.15 \mathrm{t} / \mathrm{m}^{3}$ and a coefficient of variation of $5 \%$. The friction coefficient $\mu$ is assumed to be normal distributed with mean 0.636 and a coefficient of variation of $15 \%$, see Takayama [12].

The wave direction is assumed head-on to the breakwater. The maximum significant wave height $H_{S}^{T_{L}}$ in the design lifetime $T_{L}$ is modelled as a stochastic variable on the basis of measurements with the distribution function $F_{H_{S} T_{L}}(h)=\left[1-\exp \left(-\left(\frac{h-B}{A}\right)^{k}\right)\right]^{\lambda T_{L}}$ where $\lambda$ is the average number of $H_{S}$ data values available per year. In this example $B=1.55$. To model the statistical uncertainty $k$ and $A$ are assumed to be stochastic variables. $k$ is in this example normal distributed with mean 2 and standard deviation 0.18. $A$ is normal distributed with mean 2.5 and standard deviation 0.3 (corresponding to a data sample of size 30), see Burcharth et al. [5]. Based on model tests the model uncertainty variable $Z$ in (5) and (6) is assumed normal distributed with mean 1 and standard deviation 0.2 , see van der Meer et al. [7].

The undrained shear strength of the clay $c_{u}$ is modelled by a log-Gaussian stochastic field $\left\{c_{u}(x, z)\right\}$. The mean value function and covariance function are in this paper assumed to be (with $c_{u}$ is in $\mathrm{kPa}$ and $(\mathrm{x}, \mathrm{z})$ is in meters)

$$
\begin{aligned}
E\left[c_{u}(x, z)\right] & =150+3 x \\
\operatorname{Cov}\left[c_{u}\left(x_{1}, z_{1}\right), c_{u}\left(x_{2}, z_{2}\right)\right] & =41^{2} \exp \left(-\left|\frac{x_{1}-x_{2}}{3.0}\right|\right) \exp \left(-\left(\frac{z_{1}-z_{2}}{30.0}\right)^{2}\right)
\end{aligned}
$$

\section{Reliability analysis}

With a design lifetime of $T_{L}=50$ years and a width of the caisson of $B_{r}=16.4 \mathrm{~m}$ a reliability analysis gives the results: $\beta_{\text {sliding }}=0.89, \beta_{\text {overturning }}=2.43$ and $\beta_{\text {foundation }}=0.91$. The reliability levels are seen to be rather low compared with e.g. structural systems. But compared with observed failure rates for breakwaters the reliability levels are realistic. As expected sliding and foundation failures are the critical failure modes. For sliding failure the uncertainties connected with the wave climate are dominating whereas for foundation failure the uncertainty in the shear strength of the clay is dominating.

\section{Reliability-based design}

A reliability-based optimization problem is formulated on the basis of (14)-(17). The objective function models the cost of the caisson which is assumed to be proportional to the weight of the caisson. As design variable only the width of the caisson is reasonable here. For caissons placed on rubble mounds the design variables indicated in figure 5 can be used. The lower limit on the reliability indices for the three failure modes are taken as $1.28(\approx$ a probability of failure equal to 0.1$)$. The result is an optimal $b^{*}=B_{r}=18.5$ $\mathrm{m}$ and the corresponding weight is $C^{*}=4544 \mathrm{t} / \mathrm{m}$. 


\section{Conclusions}

It is shown how reliability analysis and reliability-based design of monolithic vertical wall breakwaters can be formulated. Probabilistic models for sliding, foundation failure and overturning are described. Relevant design variables are identified and reliabilitybased optimization problems are formulated. As an illustrative example is considered a vertical wall breakwater with the caisson placed on a bedding layer and with clay in the foundation.

\section{Acknowledgements}

This work is partly supported by the Danish research program "Marin Teknik" sponsored by the Danish Technical Research Council and partly by the MAST II program "Rubble Mound Breakwater Failure Modes" sponsored by the Directorate General for Science, Research and Development of the Commision of the European Communities.

\section{References}

[1] Burcharth, H.F.: Development of a Partial Coefficient System in the Design of Rubble Mound Breakwaters. Report Subgroup F, PIANC PTC II Working group 12 on Rubble Mound Breakwaters, 1991.

[2] Goda, Y.: A New Method of Wave Pressure Calculation for the Design of Composite Breakwater. Proc. 14th Int. Conf. Coastal Eng., Copenhagen, Denmark, 1974.

[3] Goda, Y. \& T. Fukumori: Laboratory Investigation of Wave Pressures exerted upon Vertical and Composite walls. Coastal Engineering in Japan, Vol. 15, 1972, pp. 81-90.

[4] Goda, Y.: Random Seas and Design of Maritime Structures. University of Tokyo Press, 1985.

[5] Burcharth, H.F. \& J.D. Sørensen \& E. Christiani: On the Evaluation of Failure Probability of Monolithic Vertical Wall Breakwaters. Proc. "Wave Barriers in Deepwater", Port and Harbour Research Institute, Yokosuka, Japan, 1994, pp. 458-468.

[6] Takahashi, S. \& K. Tanimoto \& K. Shimosako: Dynamic Response and Sliding of Breakwater Caisson against Impulsive Breaking Wave Forces. Report of Port and Harbour Research Inst., 1994.

[7] van der Meer, J.W. \& J. Juhl \& G. Driel: Probabilistic Calculations of Wave Forces on Vertical Structures. Proc. Final Workshop, MAST G6-S Coastal Structures, Lisbon, 1992.

[8] Keaveny, J.M. \& F. Nadim \& S. Lacasse: Autocorrelation Function for Offshore Geotechnical Data. Proc. ICOSSAR89, 1989, pp. 263-270.

[9] Andersen, E.Y. \& B.S. Andreasen \& P. Ostenfeld-Rosenthat: Foundation Reliability of Anchor Block for Suspension Bridge. Proc. IFIP WG7.5, Lecture notes in Eng. Vol. 76, Springer Verlag, 1992 pp. 131-140.

[10] Enevoldsen, I. \& J.D. Sørensen: Reliability-Based Optimization in Structural Engineering. Accepted for publication in Structural Safety, 1994.

[11] Madsen, H.O., S. Krenk \& N.C. Lind: Methods of Structural Safety. Prentice-Hall, 1986.

[12] Takayama, T.: Estimation of sliding Failure Probability of present Breakwaters for Probabilistic Design. Report of Port and Harbour Research Inst., Vol. 31, No. 5, 1992. 